\title{
A Generalized Reduced Fluid Dynamic Model for Flow Fields and Electrodes in Redox Flow Batteries
}

\author{
Ziqiang Cheng ${ }^{1}$, Kevin Tenny ${ }^{2}$, Alberto Pizzolato ${ }^{1}$, Antoni Forner-Cuenca ${ }^{3}$, Vittorio \\ Verda $^{1}$, Yet-Ming Chiang ${ }^{2}$, Fikile Brushett ${ }^{2}$, and Reza Behrou ${ }^{4}$ \\ ${ }^{1}$ Politecnico di Torino \\ ${ }^{2}$ Massachusetts Institute of Technology \\ ${ }^{3}$ Eindhoven University of Technology \\ ${ }^{4}$ University of California San Diego
}

August 7, 2021

\begin{abstract}
High dimensional models typically require a large computational overhead for multiphysics applications, which hamper their use for broad-sweeping domain interrogation. Herein, we develop a modeling framework to capture the through-plane fluid dynamic response of electrodes and flow fields in a redox flow cell, generating a computationally inexpensive two-dimensional (2D) model. We leverage a depth averaging approach that also accounts for variations in out-of-plane fluid motion and departures from Darcy's law that arise from averaging across three-dimensions (3D). Our Resulting depth-averaged 2D model successfully predict the fluid dynamic response of arbitrary in-plane flow field geometries, with discrepancies of $<5 \%$ for both maximum velocity and pressure drop. This corresponds to reduced computational expense, as compared to $3 \mathrm{D}$ representations $(<1 \%$ of duration and $10 \%$ of RAM usage), providing a platform to screen and optimize a diverse set of cell geometries.
\end{abstract}

\section{Hosted file}

Manu.pdf available at https://authorea.com/users/429529/articles/533213-a-generalizedreduced-fluid-dynamic-model-for-flow-fields-and-electrodes-in-redox-flow-batteries 